\title{
CARDINALITIES OF FIRST COUNTABLE $R$-CLOSED SPACES
}

\author{
ALAN DOW AND JACK PORTER
}

\begin{abstract}
It is now well known that first countable compact Hausdorff spaces are either countable or have cardinality $c$. The situation for first countable $H$-closed spaces is that they have cardinality less than or equal $c$, and it is at least consistent that they may have cardinality $\boldsymbol{N}_{1}<c$. We show that the situation is quite different for first countable $R$-closed spaces. We begin by constructing an example which has cardinality $\boldsymbol{N}_{1}$. Let $\lambda_{0}$ be the smallest cardinal greater than $c$ which is not a successor. For each cardinal $\kappa$ with $c \leqslant \kappa \leqslant \lambda_{0}$ we construct a first countable $R$-closed space of cardinality $\kappa$. We also construct a first countable $R$-closed space of cardinality $\lambda_{0}^{\omega}$. This seems to indicate that there is no reasonable upper bound to the cardinalities of $R$-closed spaces as a function of their character.
\end{abstract}

1. Introduction. A regular space $X$ is $R$-closed if $X$ is closed in every regular space containing $X$ as a subspace, $X$ is minimal regular if $X$ has no coarser regular topology, and $X$ is strongly minimal regular (SMR) if $X$ has a base for the closed sets consisting of $R$-closed subsets. It is known that if $X$ is SMR, then $X$ is minimal regular and if $X$ is minimal regular, then $X$ is $R$-closed. A regular filter $\mathscr{F}$ on a space $X$ is a filter with the property that for $A \in \mathscr{F}$, there is an open $U \in \mathscr{F}$ such that $\operatorname{cl} U \subset A$. We shall assume that a regular filter consists of open sets. A filter $\mathscr{F}$ is free if $\cap\{\operatorname{cl} A: A \in \mathscr{F}\}=\varnothing$. An equivalent characterization of $R$-closed spaces is that there are no free regular filters, cf. [BPS].

The class of $R$-closed spaces does not seem to be a very well behaved class. For instance, not every regular space can be densely embedded in an $R$-closed space [He], and the product of $R$-closed spaces need not be $R$-closed [P]. These results are in sharp constrast to the classes of $H$-closed spaces and compact Hausdorff spaces, both of which are productive [BPS], and every Hausdorff (Tychonoff) space can be densely embedded in an $H$-closed (compact Hausdorff) space. One of the few positive results about $R$-closed spaces is in [DP1] where we show that every regular space can be embedded as a closed subspace of an $R$-closed space. This paper exhibits further distinctions between the class of $R$-closed spaces and the classes of compact Hausdorff spaces and $H$-closed spaces.

In the second section we construct an example of a first countable SMR space of cardinality $\boldsymbol{\aleph}_{1}$. A space is $\boldsymbol{\aleph}_{0}$-bounded if every countable set has compact closure. Hechler [H] has given consistent examples of first countable separable SMR spaces of cardinality $\boldsymbol{\kappa}_{1}$. However, our example requires no special set theoretic assumptions and our example is $\boldsymbol{\aleph}_{0}$-bounded. In the final section we recursively construct

Received by the editors June 22, 1981 and, in revised form, December 28, 1982.

1980 Mathematics Subject Classification. Primary 54D25; Secondary 54A25.

(C)1983 American Mathematical Society $0002-9939 / 83 \$ 1.00+\$ .25$ per page 
first countable SMR spaces for each cardinal $\kappa$ with $c \leqslant \kappa \leqslant \lambda_{0}$ and a first countable SMR space of cardinality $\lambda_{0}^{\omega}$.

A construction of $R$-closed spaces that has been put to good use by many authors is a technique due to Jones. In [DP1] we investigate this technique in some detail, but we will need a modification of it due to Stephenson [S]. We thank the referee for his or her useful suggestions.

1.1. Suppose that $X$ is a regular space containing pairwise disjoint closed sets $H_{1}$, $\mathrm{H}_{2}$ and $K$. Suppose further that we have homeomorphisms $f_{i}: H_{i} \rightarrow K$. Let $E$ be the equivalence relation on $X \times \mathbf{Z}$, where $\mathbf{Z}$ is the integers, defined by the rule $(x, i)$ $E(y, j)$ if: (i) $x=y$ and $i=j$; (ii) $x=f_{2}(y)$ and $i+1=j$, (iii) $y=f_{2}(x)$ and $j+1=i$; (iv) $x=f_{1}(y)$ and $j+1=i$; (v) $y=f_{1}(x)$ and $i+1=j$; (vi) $f_{1}(x)=f_{2}(y)$ and $i+2=j$; or (vii) $f_{1}(y)=f_{2}(x)$ and $j+2=i$. Choose two new points $\left\{P_{+}, P_{-}\right\}$ and let $D J(X)=\left\{P_{+}, P_{-}\right\} \cup(X \times \mathbf{Z}) / E$ and $J(X)=\left\{P_{+}\right\} \cup(X \times N) /\left.E\right|_{(X \times N)}$, where $N$ is the positive integers. For each $n \in N$ let $V_{n}=\left\{P_{+}\right\} \cup X \times\{m \in \mathbf{Z}$ : $m \geqslant n\}$ and $W_{n}=\left\{P_{-}\right\} \cup X \times\{m \in \mathbf{Z}: m \leqslant-n\}$. We topologize $D J(X)$ by giving $(X \times \mathbf{Z}) / E$ the quotient topology and letting $\left\{V_{n}: n \in N\right\}$ and $\left\{W_{n}: n \in N\right\}$ form neighborhood bases for $P_{+}$and $P_{-}$respectively. With this topology $D J(X)$ is a regular space, and we let $J(X)$ have the subspace topology.

Proposition 1.2. Let $X$ be locally compact and Hausdorff, and let $H_{1}, H_{2}, K, f_{1}$ and $f_{2}$ be as in 1.1. Suppose that for some $n \in \omega$ and any sequence $U_{0}, \ldots, U_{n+1}$ with cl $U_{i+1} \subset$ int $\mathrm{cl} U_{i}$ for each $i \leqslant n, \operatorname{cl} U_{n+1}$ not compact implies that $\operatorname{cl} U_{0} \cap K$ is not compact. Then DJ $(X)$ is $S M R$ and $J(X)$ is R-closed.

Proof. Since $X$ is locally compact, so is $D J(X) \backslash\left\{P_{+}, P_{-}\right\}$. By 1.2 of [DP1] and the fact that for each $m \in N, V_{m}$ and $W_{m}$ are homeomorphic to $J(X)$, it suffices to show that $J(X)$ is $R$-closed.

Suppose that $\mathscr{F}$ is a free regular filter on $J(X)$. Since $\mathscr{F}$ is free, there is a $U_{0} \in \mathscr{F}$ such that $P_{+} \notin \operatorname{cl} U_{0}$. Suppose that $k \in N$ is such that $V_{k} \cap \operatorname{cl} U_{0}=\varnothing$. Let $m=$ $k \cdot(n+1)$ and recursively choose $\left\{U_{i}: i \leqslant m\right\} \subseteq \mathscr{F}$ such that $\operatorname{cl} U_{i+1} \subset U_{i}$ for $i=0,1, \ldots, m-1$. Since $\mathscr{F}$ is free, for some $i<m, \operatorname{cl} U_{m} \cap X \times\{i\}$ is not compact. Therefore, by assumption on $X, \operatorname{cl} U_{m-(n+1)} \cap K \times\{i\}$ is not compact. Since $K \times\{i\}$ is identified with $H_{2} \times\{i+1\}$ by $f_{2}$, we have that $\mathrm{cl} U_{m-(n+1)} \cap X \times\{i+1\}$ is not compact. By induction, this implies that $\operatorname{cl} U_{m-m} \cap X \times\{k\}$ is not compact; however, this contradicts that $\operatorname{cl} U_{0} \cap V_{k}=\varnothing$.

2. The $\boldsymbol{\aleph}_{1}$ example. Let us begin by establishing some notation. Let $\Lambda=\left\{\lambda_{\alpha}\right.$ : $\left.\alpha<\omega_{1}\right\}$ be an order preserving listing of the limit ordinals less than $\omega_{1}$. For each $n \in \omega$, let $A_{n}=\left[\left(\omega_{1} \backslash \Lambda\right) \times(n+1) \cup \Lambda\right] \times\{n\}$, and let $X=\cup_{n \in \omega} A_{n}$. Recall that an ordinal is the set of its predecessors. For each $\alpha \in \omega_{1}$, let

$$
X_{\alpha}=\bigcup_{n \in \omega}\left[\lambda_{\alpha} \backslash \Lambda \times(n+1) \cup\left\{\lambda_{\beta}: \beta \leqslant \alpha\right\}\right] \times\{n\}
$$

We will construct, for $\alpha \in \omega_{1}$, a topology $\tau_{\alpha}$ on $X_{\alpha}$ which is compact Hausdorff and has a basis of compact open sets. This construction is a modification of Vaughan's [V] construction of a first countable, countably compact, nonnormal 
space and is indeed itself such a space. We have not been able to show that Vaughan's space satisfies the hypothesis of 1.2 and suspect it does not as it contains countably many pairwise disjoint clopen copies of $\omega_{1}$ whose union is dense.

To help clarify the construction we give an informal description first. Each column $A_{n}$ is $\omega_{1} \times(n+1)$ with the limit ordinals identified, and will be homeomorphic to $\omega_{1}$. We think of each column $A_{n} \backslash(\Lambda \times\{n\})$ as containing $n+1$ columns. If we take a horizontal cross section at level $\alpha \in \omega_{1} \backslash \Lambda$ by choosing the element out of the $k$ th column of $A_{n}$ for each $n>k$ this sequence will converge to the point in the $k$ th column of $A_{k}$. The limit levels will compactify what is below them with, in addition, $\{(\lambda, n): 1 \leqslant n<\omega\}$ converging to $(\lambda, 0)$.

Suppose that $\alpha \in \omega_{1}$ and for $\beta<\alpha$ we have a constructed a Hausdorff topology $\tau_{\beta}$ on $X_{\beta}$ such that

(a) $\tau_{\beta}$ has a countable base of compact open sets;

(b) for each $n \in \omega, \gamma \in \lambda_{\beta} \backslash \Lambda$ and $k<n$ the point $(\gamma, k, n)$ is isolated;

(c) for each $k \in \omega$ and $\gamma \in \lambda_{\beta} \backslash \Lambda$, a neighborhood base for $(\gamma, k, k)$ is $\{\{(\gamma, k, k)\} \cup\{(\gamma, k, m): m>n\}: k \leqslant n<\omega\}$

(d) for each $\delta \leqslant \beta$ and $n<\omega$ the set $\left\{(\gamma, k, n): \gamma<\lambda_{\delta}, k \leqslant n\right\} \cup\left\{\left(\lambda_{\delta}+1 \cap \Lambda\right)\right.$ $\times\{n\}\}$ is compact;

(e) for each $\delta \leqslant \beta,\left\{\left(\lambda_{\delta}, n\right)\right.$ : $\left.0<n<\omega\right\}$ converges to $\left(\lambda_{\delta}, 0\right)$; and

(f) $\left(X_{\delta}, \tau_{\delta}\right)$ is an open subspace of $\left(X_{\beta}, \tau_{\beta}\right)$ for each $\delta<\beta$.

We will define $\tau_{\alpha}$ by defining neighborhood bases at each of the points of $X_{\alpha} \backslash \cup_{\beta<\alpha} X_{\beta}$.

Step 1. For each $\gamma \in \lambda_{\alpha} \backslash \cup_{\beta<\alpha} \lambda_{\beta}+1$ and $k<n<\omega$, we put $\{(\gamma, k, n)\}$ in $\tau_{\alpha}$.

Step 2. For each $\gamma \in \lambda_{\alpha} \backslash \cup_{\beta<\alpha} \lambda_{\beta}+1$ and $k<\omega$, a neighborhood base for $(\gamma, k, k)$ will be

$$
\{\{(\gamma, k, k)\} \cup\{(\gamma, k, m): m>n\}: k \leqslant n<\omega\} .
$$

Step 3. Let $\left\{\gamma_{i}: i \in \omega\right\} \subset \lambda_{\alpha} \backslash \Lambda$ be a strictly increasing sequence converging to $\lambda_{\alpha}$. For each $0<n<\omega$, let $B_{0}(n)=\left[\left(\gamma_{0} \backslash \Lambda\right) \times(n+1) \cup\left(\Lambda \cap \gamma_{0}\right)\right] \times\{n\}$ and recursively define, for $1 \leqslant i<\omega$,

$$
B_{i}(n)=\left[\left(\gamma_{i} \backslash \Lambda\right) \times(n+1) \cup\left(\Lambda \cap \gamma_{i}\right)\right] \times\{n\} \backslash B_{i-1}(n) .
$$

It is easily seen that for each $n \in \omega \backslash\{0\},\left\{B_{i}(n): i \in \omega\right\}$ is locally finite in $X_{\alpha} \backslash\left(\left\{\lambda_{\alpha}\right\} \times \omega\right)$. In addition, by induction, assumptions (c), (d), and (f), each $B_{i}(n)$ is a compact set. Each point of $B_{i}(n)$ has a neighborhood base of compact open sets already defined. By the definition of these neighborhood bases we may choose, for each $n>0$, a locally finite collection of compact open subsets, $\left\{C_{i}(n): i \in \omega\right\}$, of $X_{\alpha} \backslash\left(\left\{\lambda_{\alpha}\right\} \times \omega\right)$ so that $C_{i}(n) \cap A_{n}=B_{i}(n)$ and $C_{i}(n) \cap A_{j}=\varnothing$ for $j \leqslant \max \{i, n\}$, $j \neq n$. Recursively define for $1 \leqslant n<\omega$ a neighborhood base at $\left(\lambda_{\alpha}, n\right)$ as follows: For each $j<\omega$,

$W_{j}\left(\left(\lambda_{\alpha}, n\right)\right)=\left[\left\{\left(\lambda_{\alpha}, n\right)\right\} \cup \cup\left\{C_{i}(n): j<i<\omega\right\}\right] \backslash \cup\left\{W_{n}\left(\left(\lambda_{\alpha}, m\right)\right): 0<m<n\right\}$.

Note that for $m<n, W_{n}\left(\left(\lambda_{\alpha}, m\right)\right) \cap A_{n}=\varnothing$ and that it can be shown by induction on $n$ that $W_{i}\left(\left(\lambda_{\alpha}, n\right)\right)$ is compact open. To check (d), observe that for any $j \in \omega$, $\left(A_{n} \cap X_{\alpha}\right) \backslash W_{j}\left(\left(\lambda_{\alpha}, n\right)\right)=\bigcup\left\{B_{i}(n): i \leqslant j\right\}$ which is compact. 
Step 4. By construction $X_{\alpha} \backslash\left\{\left(\lambda_{\alpha}, 0\right)\right\}$ has a countable base of compact open sets. We let $X$ be the one point compactification of $X_{\alpha} \backslash\left\{\left(\lambda_{\alpha}, 0\right)\right\}$. This clearly satisfies conditions (e) and (f) and the others obviously hold by construction.

We let $\tau$ be the topology on $X$ generated by the open base $\cup_{\alpha<\omega_{1}} \tau_{\alpha}$. With this topology $X$ is first countable, zero dimensional (has a base of clopen sets), locally compact, $\boldsymbol{\aleph}_{0}$-bounded, nonnormal and has cardinality $\boldsymbol{\aleph}_{1}$. To see that $X$ is $\boldsymbol{\aleph}_{0^{-}}$. bounded we simply note that any countable set is contained in some $X_{\alpha}$. Nonnormality will be a trivial consequence of the following.

Let $U$ be an open set containing a closed noncompact subset of $A_{n}$, where $n \in \omega$. We will show that $\mathrm{cl} U \cap A_{0}$ is not compact. Since $A_{n}$ is homeomorphic to $\omega_{1}, A_{n} \backslash U$ is countable [GJ]. Therefore there is an $\alpha<\omega_{1}$ such that for $\gamma>\alpha$ and $\gamma \notin \Lambda$, $(\gamma, n, n) \in U$. For each such $\gamma$, there is a $k \in \omega$ such that $\{(\gamma, n, j): j>k\} \subset U$ since $U$ is open. It follows that there are an uncountable set $\Gamma \subset \omega_{1} \backslash \Lambda$ and a $k \in \omega$ such that for each $\gamma \in \Gamma,\{(\gamma, n, j): j>k\} \subset U$. Now, for each limit $\lambda$ of $\Gamma$ and $j>k,(\lambda, j) \in \operatorname{cl} U$ since $(\lambda, j) \in \operatorname{cl}\{(\gamma, n, j): \gamma \in \Gamma\}$. Therefore $(\lambda, 0) \in \operatorname{cl} U$ for each limit $\lambda$ of $\Gamma$. Since $\Gamma$ is uncountable, $\operatorname{cl} U \cap A_{0}$ is not compact.

Now since each $A_{n}$ is homeomorphic to $\omega_{1}$ if we let $H_{1}=A_{1}, H_{2}=A_{2}$ and $K=A_{0}$, we have that $X$ satisfies the conditions of 1.2 with $n=0$. We have shown

THEOREM 2.1. DJ $X)$ is a first countable SMR space with cardinality $\boldsymbol{\aleph}_{1}$.

3. Cardinals greater than $c$. In this section we will construct first countable SMR spaces with cardinalities up to $\lambda_{0}$ where $\lambda_{0}$ is the first singular cardinal greater than c. Assuming GCH, $\lambda_{0}=\boldsymbol{\aleph}_{\omega}$. We will make extensive use of a space developed by Mrowka and Isbell then modified by Stephenson [S] and Hechler [H].

3.1. Suppose $D$ is an infinite discrete set and $R$ is a family of infinite pairwise almost disjoint countable subsets of $D$. ( $A$ and $B$ are almost disjoint if $|A \cap B|<\omega$.) We will give $X=D \cup R$ the following topology. $D$ is open and discrete while a neighborhood base for $r \in R$ is $\{\{r\} \cup r \backslash F: F$ is a finite subset of $r\}$. It is easily shown that $X$ is a first countable locally compact Hausdorff space.

Suppose that $R$ contains disjoint subsets $H, K$ with $H \cup K=R$ and $|H|=|K|$. Suppose further that there is an $n \in \omega$ such that for any sequence $U_{0}, \ldots, U_{n+1} \subset D$ with $\operatorname{cl} U_{i+1} \subset$ int $\operatorname{cl} U_{i}$ for $i \leqslant n,\left|\operatorname{cl} U_{n+1} \cap H\right| \geqslant \aleph_{0}$ implies $\left|\operatorname{cl} U_{0} \cap K\right|>\boldsymbol{\aleph}_{0}$. It is then clear that if we let $H_{1} \cup H_{2}=H$ with $\left|H_{1}\right|=\left|H_{2}\right|$ and $f_{i}$ be any isomorphism from $H_{i}$ to $K$ for $i=1,2$, then $X$ satisfies the conditions 1.2. Therefore $D J(X)$ would be SMR. This will be our plan, to recursively construct $X_{\kappa}$, for $c \leqslant \kappa \leqslant \lambda_{0}$, so that $X_{\kappa}=D_{\kappa} \cup R_{\kappa}$ with $\left|R_{\kappa}\right|=\kappa$ and $R_{\kappa}$ satisfying the above.

To begin we borrow a result by Hechler $[\mathbf{H}]$ whose purpose was to remove the assumption of the contiuum hypothesis from an example by Stephenson [S].

3.2. THEOREM. There is a maximal almost disjoint family $R_{c}$ of infinite subsets of $\omega$ such that $\left|R_{c}\right|=c$ and there is a subset $H_{c} \subset R_{c}$ with $\left|H_{c}\right|=c$ such that for $U \subset \omega$, $\left|\left\{r \in H_{n}:|U \cap r|=\boldsymbol{\aleph}_{0}\right\}\right| \geqslant \boldsymbol{\aleph}_{0}$ implies $\left|\left\{r \in R_{c} \backslash H_{r}:|U \cap r|=\boldsymbol{\aleph}_{0}\right\}\right|=c$.

For a space $X=D \cup R$ as in 3.1 we will say that $(X, H, K)$ satisfies $\left({ }_{n}\right)$ if the following hold: (i) $H \subset R$ and $K=R \backslash H$; (ii) $|H|=|K|=|R|$; and (iii) for 
$U_{0}, \ldots, U_{n+1} \subseteq D$ with $\operatorname{cl} U_{i+1} \subset$ int $\operatorname{cl} U_{i}$ for $i \leqslant n$, then $\left|\operatorname{cl} U_{n+1} \cap H\right| \geqslant \boldsymbol{\aleph}_{0}$ implies $\left|\operatorname{cl} U_{0} \cap K\right|>\boldsymbol{\kappa}_{0}$. Let $D_{c}=\omega$ and let $R_{c}$ and $H_{c}$ be as in 3.2. Then $X_{c}=D_{c} \cup R_{c}$ with the topology as in 3.1 satisfies $\left(*_{0}\right)$. Indeed, for $r \in R_{c}$ and $W \subset D_{c}, r \in \operatorname{cl} W$ iff $|r \cap W|=\boldsymbol{\aleph}_{0}$. Let $K_{c}=R_{c} / H_{c}$.

Therefore if $U_{0}, U_{1} \subset D_{c}$ are as in (iii) with $\left|\operatorname{cl} U_{1} \cap H_{c}\right| \geqslant \boldsymbol{\aleph}_{0}$ then by 3.2 $\left|\operatorname{cl} U_{1} \cap K_{c}\right|=c$. Hence $\left|\operatorname{cl} U_{0} \cap K_{c}\right|>\aleph_{0}$.

3.3. The main construction. Let $\kappa$ be a cardinal such that $\kappa^{\omega}=\kappa$. Suppose that $X_{\kappa}=D_{\kappa} \cup R_{\kappa}$ is as in 3.1 with $\left|D_{\kappa}\right| \leqslant\left|R_{\kappa}\right|=\kappa$. Suppose also that $n \in \omega$ and that $\left(X_{\kappa}, H_{\kappa}, K_{\kappa}\right)$ satisfy $\left(*_{n}\right)$. We shall let $D_{\kappa^{+}}=D_{\kappa} \times \kappa^{+}$and define an almost disjoint family, $R_{\kappa^{+}}$, of countable subsets of $D_{\kappa^{+}}$and subsets $H_{\kappa^{+}}$and $K_{\kappa^{+}}$of $R_{\kappa^{+}}$so that $\left(X_{\kappa^{+}}, H_{\kappa^{+}}, K_{\kappa^{+}}\right)$satisfies $\left(*_{n+1}\right)$.

An ordinal $\delta$ is said to have countable cofinality, cf $\delta=\omega$, if there is a sequence $\left\{\gamma_{n}: n \in \omega\right\} \subseteq \delta$ such that $\delta=\sup \left\{\gamma_{n}\right\}$. For each $\delta<\kappa^{+}$with cf $\delta=\omega$ we shall construct a set $R(\delta)$ as follows. Choose any strictly increasing sequence $\left\{\gamma_{n}: n \in \omega\right\}$ converging to $\delta$. Let $R(\delta)$ be a maximal family of almost disjoint infinite countable subsets of $D_{\kappa} \times \delta$ such that for each $r \in R(\delta)$ and $n \in \omega,\left|r \cap D_{\kappa} \times \gamma_{n}\right|<\boldsymbol{\aleph}_{0}$. Note that $|R(\delta)| \leqslant\left|D_{\kappa} \times \delta\right|^{\omega} \leqslant \kappa^{\omega}=\kappa$. Fix an injection $f_{\delta}$ from $R(\delta)$ into $H_{\kappa}$. We define $R_{\kappa^{+}}=\left\{r \cup\left(f_{\delta}(r) \times\{\delta\}\right): \delta<\kappa^{+}\right.$with cf $\delta=\omega$ and $\left.r \in R(\delta)\right\} \cup\{r \times\{\gamma\}: \gamma<$ $\kappa^{+}, r \in R_{\kappa}$, and if cf $\left.\gamma=\omega, r \notin f_{\gamma}(R(\gamma))\right\}$. Each element of $R_{\kappa^{+}}$is a countable subset of $D_{\kappa^{+}}$and $R_{\kappa^{+}}$is an almost disjoint family. Let us define $H_{\kappa^{+}}=\left\{r \in R_{\kappa^{+}}\right.$: there are an $r_{1} \in H_{\kappa}$ and $\gamma<\kappa^{+}$with $\left.r_{1} \times\{\gamma\} \subset r\right\}$. Also let $K_{\kappa^{+}}=R_{\kappa^{+}} \backslash H_{\kappa^{+}}=\{r$ $\in R_{\kappa^{+}}$: there are an $r_{1} \in K_{\kappa}$ and $\gamma<\kappa^{+}$with $\left.r_{1} \times\{\gamma\}=r\right\}$. Note that $\left|H_{\kappa^{+}}\right|=\left|H_{\kappa}\right|$ $\cdot \kappa^{+}=\left|K_{\kappa}\right| \cdot \kappa^{+}=\left|K_{\kappa^{+}}\right|$. Let us check that $\left(X_{\kappa^{+}}, H_{\kappa^{+}}, K_{\kappa^{+}}\right)$satisfies $\left({ }_{n+1}\right)$. Let $U_{0}, \ldots, U_{n+2} \subset D_{\kappa}$ with cl $U_{i+1} \subset$ int cl $U_{i}$; for $i \leqslant n+1$ and $\left|\operatorname{cl} U_{n+2} \cap H_{\kappa}\right| \geqslant \kappa_{0}$. Suppose first that there is a $\gamma<\kappa^{+}$such that $\operatorname{cl} U_{n+2} \cap\left\{r \in H_{\kappa}+\right.$ : there is an $r_{1} \in H_{\kappa}+$ with $\left.r_{1} \times\{\gamma\} \subset r\right\}$ is infinite. Therefore, since $\left(X_{\kappa}, H_{\kappa}, K_{\kappa}\right)$ satisfies $\left(*_{n}\right)$, $\operatorname{cl} U_{0} \cap\left\{r \in K_{\kappa}\right.$ : there is an $r_{1} \in K_{\kappa}$ with $\left.r_{1} \times\{\gamma\}=r\right\}$ is uncountable. Hence $\left|\mathrm{cl} U_{0} \cap K_{\kappa}\right|>\aleph_{0}$. On the other hand, suppose that there is an infinite increasing sequence $\left\{\gamma_{j}: j \in \omega\right\} \subset \kappa^{+}$such that, for each $j \in \omega$, there are an $r_{1}(j) \in H_{\kappa}$ and an $r(j) \in H_{\kappa} \cap \cap \mathrm{cl} U_{n+2}$ with $r_{1}(j) \times\left\{\gamma_{j}\right\} \subset r(j)$. Therefore, for each $j \in \omega, U_{n+2} \cap$ $D_{\kappa} \times\left(\gamma_{j}+1\right)$ is infinite. So let $\delta=\sup \left\{\gamma_{j}: j \in \omega\right\}$ and choose an infinite set $S \subset R(\delta)$ such that $s \cap U_{n+2}$ is infinite for each $s \in S$. This can be done since $s \cap D_{\kappa} \times \gamma_{j}$ is finite for each $j \in \omega$ and $s \in R(\delta)$. Therefore, $s \cup\left(f_{\delta}(s) \times\{\delta\}\right) \in$ $\operatorname{cl} U_{n+2}$ for each $s \in S$ and by the above $\mid \operatorname{cl} U_{0} \cap K_{\kappa}+>\boldsymbol{\aleph}_{0}$.

We now obtain the following results.

THEOREM 3.4. For each cardinal $\kappa$ with $c \leqslant \kappa<\lambda_{0}$ there is a first countable SMR space with cardinality $\kappa$.

Proof. Observe that for a cardinal $\kappa$ with $\operatorname{cf}(\kappa)>\omega, \kappa^{\omega}=\Sigma\left\{\alpha^{\omega}: \alpha<\kappa\right\}$. It follows that $\left(c^{+}\right)^{\omega}=\Sigma\left\{\left|\alpha^{\omega}\right|: \alpha<c^{+}\right\}=c^{+} \cdot c=c^{+}$. Similarly, by induction, $\kappa^{\omega}=$ $\kappa$ for $c \leqslant \kappa<\lambda_{0}$. Therefore, by 3.2 and 3.3, we have a space $X_{\kappa}$ of cardinality $\kappa$ such that $\left(X_{\kappa}, H_{\kappa}, K_{\kappa}\right)$ satisfies $\left({ }_{n}\right)$ for some $n \in \omega$. We partition $H_{\kappa}$ into two sets $H_{1}$ and $H_{2}$ with $\left|H_{1}\right|=\left|H_{2}\right|$. By $1.2, D J\left(X_{\kappa}\right)$ is a first countable SMR space.

The following result follows easily from the results in $[P]$. 
THEOREM 3.5. A product of first countable SMR spaces is itself SMR.

COROLlaRY 3.6. There are first countable, SMR spaces of cardinality $\lambda_{0}$ and $\lambda_{0}^{\omega}$, respectively.

Proof. For $n \in \omega$, let $\kappa_{n}$ be the $n$th successor of $c$. Thus, $\lambda_{0}=\Sigma\left\{\kappa_{n}: n \in \omega\right\}$. For each $n \in \omega$, let $X_{n}$ be a first countable, SMR space of cardinality $\kappa_{n}$. Let $Y$ be the topological sum of $\left\{X_{n}: n \in \omega\right\}$ (we write $Y=\cup\left\{X_{n}: n \in \omega\right\}$ ) and $X=\left\{P_{+}\right\} \cup Y$. Now, $U \subseteq X$ is defined to be open if $U \cap Y$ is open in $Y$ and if $P_{+} \in U$, there is $m \in \omega$ such that $U \supseteq \cup\left\{X_{n}: n \geqslant m\right\}$. It is immediate that $X$ is first countable and $R$-closed and has cardinality $\lambda_{0}$. Since each $X_{n}$ is SMR, then $X$ is also SMR. Finally let $Z=\Pi\left\{X_{n}: n \in \omega\right\}$. By $3.5, Z$ is SMR and is clearly first countable. The cardinality of $Z$ is $\left|\Pi\left\{X_{n}: n \in \omega\right\}\right|=\lambda_{0}^{\omega}$.

\section{REFERENCES}

[BPS] M. P. Berri, J. R. Porter and R. M. Stephenson, Jr., A survey of minimal topological spaces, General Topology and its Relations to Modern Analysis and Algebra, III (Proc. Conf., Kanpur, 1968), Academia, Prague, 1971, pp. 93-114.

[DP1] A. Dow and J. Porter, Embedding in R-closed spaces, Topology Appl. 15 (1983), 45-58.

[DP2] _ C Cardinalities of $H$-closed spaces, Topology Proc. 7 (1982).

[G] A. A. Gryzlow, Two theorems on the cardinality of topological spaces, Soviet Math. Dokl. 21 (1980), 506-509.

[H] S. H. Hechler, Two R-closed spaces revisited, Proc. Amer. Math. Soc. 56 (1976), 303-309.

[He] H. Herrlich, $T_{\nu}$-Abgeschlossenheit und $T_{\nu}$-Minimalität, Math. Z. 88 (1965), 285-294.

[P] D. Pettey, Products of regular-closed spaces, Topology Appl. 14 (1982), 189-199.

[S] R. M. Stephenson, Jr., Two R-closed spaces, Canad. J. Math. 24 (1972), 286-292.

[V] J. E. Vaughan, A countably compact, first countable, non-normal $T_{2}$-space, Proc. Amer. Math. Soc. 15 (1979), 339-342.

Department of Mathematics, University of Kansas, Lawrence, Kansas 66045 (Current address of Jack Porter)

Current address (Alan Dow): Department of Mathematics, University of Toronto, Toronto, Ontario, Canada M5S 1Al 